KUNS-1304

$\mathrm{HE}$ (TH) $94 / 16$

SU-4240-592

hep-ph/9505206

\title{
Solving the Homogeneous Bethe-Salpeter Equation
}

\author{
Masayasu Harada $a^{a, v^{\prime}}$ and Yuhsuke Yoshida $a^{b, 巾^{\dagger}}$ \\ a Department of Physics, Syracuse University \\ Syracuse, NY 13244-1130, USA \\ $b$ Department of Physics, Kyoto University \\ Kyoto 606-01, Japan
}

May 1, 1995

\begin{abstract}
We study a method for solving the homogeneous Bethe-Salpeter equation. By introducing a 'fictitious' eigenvalue $\lambda$ the homogeneous BetheSalpeter equation is interpreted as a linear eigenvalue equation, where the bound state mass is treated as an input parameter. Using the improved ladder approximation with the constant fermion mass, we extensively study the spectrum of the fictitious eigenvalue $\lambda$ for the vector bound states and find the discrete spectrum for vanishing bound state mass. We also evaluate the bound state masses by tuning appropriate eigenvalues $\lambda$ to be unity, and find massless vector bound states for specific values of the constant fermion masses.
\end{abstract}

* e-mail address : mharada@npac.syr.edu

$\dagger$ e-mail address : yoshida@gauge.scphys.kyoto-u.ac.jp 


\section{Introduction}

Bethe-Salpeter (BS) equations [1], 2, 3] for the fermion-antifermion systems in gauge theories are important for studying the properties of positronium, mesons and so on. The BS equation in the improved ladder approximation was applied to the bound state problem in QCD. Especially, the numerical solution for the massless pion was given in refs. [4, 5], and they succeeded to reproduce the property of the chiral symmetry in the light quark sector. Moreover, the BS equation for the $B$ meson was solved in the heavy quark limit.[6]

Let us explain the solvability of the homogeneous BS equation for the pion case. The reason is essentially found in the Nambu-Goldstone's theorem[7, []. First of all, the pion is known to be massless without solving the BS equation. Moreover, thanks to the axial Ward-Takahashi identity, one scalar component, $\widehat{S}$, of the pion BS amplitude is found to be identical with the mass function of the quark. Then, the other components are obtained by solving the resultant inhomogeneous equation, where the component $\widehat{S}$ is treated as an inhomogeneous term and linear algebraic techniques are applicable.

The reason why the homogeneous BS equation for the $B$ meson can be solved is that the equation in the heavy quark limit becomes an eigenvalue equation. The BS amplitude and the binding energy of the $B$ meson are corresponding to the eigenvector and the eigenvalue, respectively. Thus, the problem is reduced to solving the eigenvalue equation, which can be done by linear algebraic techniques.

How about other cases? Generally, there are two problems for solving the homogeneous BS equation for the massive bound state:

i) We need the fermion propagator in solving the BS equation. It is natural to take account of the quantum corrections to the fermion propagator. Actually this is required by the consistency with the chiral Ward-Takahashi identity 9, 10, 11] in QCD. We have to work in the time-like region for the center-of-mass momentum $q^{\mu}$ (on-shell), while we carry out the Wick rotation to the relative momentum $p^{\mu}$ (off-shell). In a certain gauge choice, the fermion propagator in the homogeneous BS equation takes the form $i S_{F}^{-1}(p \pm q / 2)=\not p \pm \not / 2-$ $\Sigma\left(-(p \pm q / 2)^{2}\right)$, where $q^{2}$ is identical to the bound state mass squared and the time component $p^{0}$ is pure imaginary. The momenta flowing along the fermion and antifermion lines become complex. Thus, we need the mass function $\Sigma(z)$ on the complex plane.

ii) The homogeneous BS equation depends on the bound state mass complicatedly. 
It is not any linear eigenvalue equation so that it seems impossible to obtain the bound state mass and its corresponding BS amplitudes directly. Even when we adopt the constant mass approximation for the mass function, the complexity is not avoided. The homogeneous BS equation includes both the linear and quadratic terms of the bound state mass simultaneously.

One way to avoid the above two problems is using the inhomogeneous BS equation in space-like region. 12, 13 In general, the solution of the inhomogeneous BS equation has the perturbative expansion series which begins with the inhomogeneous part. The inhomogeneous BS equation may be solved by the iterations: the inhomogeneous part is taken as a first trial solution, and is substituted into the inhomogeneous BS equation in order to make more accurate solution. Repeating this iteration many times, we obtain the solution with appropriate accuracy.

Another way is taking further approximation in addition to the ladder approximation. When we take the BS kernel to be the instantaneous (Coulomb-like) interaction, the homogeneous BS equation reduces to the Salpeter equation. [14] On the other hand, in ref. [15] the authors work for the Euclidean total momentum and solve the BS equation by expanding the BS amplitude in terms of $S O(4)$ orthogonal Tschebyshev polynomials and dropping higher order polynomials. They investigate various mesons with their approximation. 16]

Now, let us ask the following question: "How do we solve the above two problems i) and ii) to obtain solutions of the homogeneous BS equation?" As for the problem i), the Schwinger-Dyson (SD) equation on the complex plane was solved to obtain the mass function. 17, 18] This analytic continuation is also done using the SD equation in the integral form. [In appendix A, following ref. [19], we briefly review how to derive the fermion mass function on the complex plane.] Then we concentrate our attention on the problem ii).

Traditionally, the BS equation is solved by regarding a coupling constant as an eigenvalue and the bound state mass as an input.[3] In this paper, to solve the homogeneous BS equation for a given coupling constant numerically, we introduce a 'fictitious' eigenvalue $\lambda$ by replacing the BS kernel $K$ with $\lambda K$. We notice that it is corresponding to replacing the coupling $\alpha$ with $\lambda \alpha$ in the (improved) ladder and constant fermion mass approximations. By adjusting the input bound state mass so as to set the fictitious eigenvalue $\lambda$ equal to unity, we get masses of the bound states. As stated above, it is natural to use the full propagator which is determined by the SD equation in the same approximation as that for the BS equation. But here we concentrate our attention on the numerical method to solve the homogeneous BS 
equation, and we adopt the constant fermion mass approximation.

In many literatures [20], by regarding the coupling constant as an eigenvalue, the $\mathrm{BS}$ equation is solved for the fermion-antifermion bound state in the fixed coupling case. The behavior of the 'eigenvalue' $\alpha$, which corresponds to our fictitious eigenvalue $\lambda$, is studied in detail. For applying to QCD we should use the running coupling 21, 22], but we do not know the behavior of the fictitious eigenvalue $\lambda$. Then, before calculating the bound state mass, we study the behavior of $\lambda$ relating it with the norms of BS amplitudes. Moreover, we check the existence of the discrete spectrum: our choice of the running coupling 13 becomes a constant in the low energy region and the low energy behavior of the theory would be similar to that of the strong coupling QED. For a type of BS equation in the strong coupling QED, it is shown 20, 23] that there is no discrete spectrum.

After studying the behavior of $\lambda$, we solve the original BS equation for the vector bound state numerically, and obtain the bound state mass. As an instructive example to check the validity of our method, we also calculate the spectrum of the orthopositronium, which is a vector bound state of electron and positron.

This paper is organized as follows. In section 2 we review the homogeneous BS equation. The BS amplitude is defined and is expanded in terms of the invariant amplitudes. The normalization condition for the BS amplitude is given. Section 3 is devoted to explanation of the method for solving the homogeneous BS equation. We extensively study the behavior of the fictitious eigenvalue $\lambda$ in the running coupling case in section 4.1. In section 4.2, as a consistency check we study the positronium in the weak coupling QED, and we calculate the bound state masses in the running coupling case. The discussions are found in section 5 .

\section{Homogeneous Bethe-Salpeter Equation}

In this section we show the basic formulations in solving the homogeneous BetheSalpeter (HBS) equation for vector bound state. Our formulations are rather transparent especially for the vector case.

\subsection{BS Amplitude}

Let us express the fermion-antifermion bound state of the vector type $\left(J^{P C}=1^{--}\right)$ as $|V(q, \epsilon)\rangle$ where $q_{\mu}$ is the momentum of the bound state and $\epsilon_{\mu}$ is the polarization 
vector. $\left[\epsilon \cdot q=0, \epsilon^{2}=-1\right.$.] The $\mathrm{BS}$ amplitude $\chi(p ; q, \epsilon)$ is defined by

$$
\langle 0|\mathrm{~T} \psi(x) \bar{\psi}(y)| V(q, \epsilon)\rangle=e^{-i q X} \int \frac{d^{4} p}{(2 \pi)^{4}} e^{-i p r} \chi(p ; q, \epsilon)
$$

where $X^{\mu}$ and $r^{\mu}$ are the center-of-mass coordinate and the relative coordinate, respectively:

$$
X^{\mu}=\frac{x^{\mu}+y^{\mu}}{2}, \quad r^{\mu}=x^{\mu}-y^{\mu} .
$$

The BS amplitude is bispinor and is defined only on the mass-shell of the bound state $q^{2}=M_{V}^{2}$, where $M_{V}$ is the mass of the bound state. We usually perform the Wick rotation to the $\mathrm{BS}$ amplitude, and the time component of the relative momentum $p_{\mu}$ becomes pure imaginary, while the quantities $q_{\mu}$ and $\epsilon_{\mu}$ are Minkowski vectors and real.

The BS amplitude of the vector bound state is expanded by eight invariant amplitudes $\chi^{1}, \cdots, \chi^{8}$ :

$$
\chi(p ; q, \epsilon)=\sum_{i=1}^{8} \Gamma_{i}(p ; q, \epsilon) \chi^{i}(p ; q) .
$$

We note that the dependence of the polarization vector $\epsilon_{\mu}$ is isolated in the bispinor base $\Gamma_{i}(p ; q, \epsilon)$. The following choice of the bispinor bases $\Gamma_{i}(p ; q, \epsilon)(i=1, \cdots, 8)$ are convenient:

$$
\begin{aligned}
& \Gamma_{1}=\not, \quad \Gamma_{2}=\frac{1}{2}[\notin, \not p](p \cdot \widehat{q}), \quad \Gamma_{3}=\frac{1}{2}[\notin, \widehat{q}], \quad \Gamma_{4}=\frac{1}{3 !}[\not, \not p, \widehat{q}], \\
& \Gamma_{5}=(\epsilon \cdot p), \quad \Gamma_{6}=\not p(\epsilon \cdot p), \quad \Gamma_{7}=\not{\not}(p \cdot \widehat{q})(\epsilon \cdot p), \quad \Gamma_{8}=\frac{1}{2}[\not p, \not{\not}](\epsilon \cdot p),
\end{aligned}
$$

where $\widehat{q}_{\mu}=q_{\mu} / M_{V}$ and $[a, b, c] \equiv a[b, c]+b[c, a]+c[a, b]$. The invariant amplitude $\chi^{i}(p ; q)$ is a scalar function in $p^{2}$ and $p \cdot q$. . So, after the Wick rotation, it is convenient to introduce the real variables $u$ and $x$ by

$$
p \cdot q=i M_{V} u, \quad p^{2}=-u^{2}-x^{2} .
$$

The time component of $p^{\mu}$ is $i u$ and the magnitude of the spatial components of $p^{\mu}$ is $x$ in the rest frame $q^{\mu}=\left(M_{V}, \overrightarrow{0}\right)$. The invariant amplitude is a function in $u$ and $x ; \chi^{i}=\chi^{i}(u, x)$. By the charge conjugation properties

$$
\begin{aligned}
C \chi(-p ; q, \epsilon)^{T} C^{-1} & =-\chi(p ; q, \epsilon), \\
C \Gamma_{i}(-p ; q, \epsilon)^{T} C^{-1} & =-\Gamma_{i}(p ; q, \epsilon),
\end{aligned}
$$

\footnotetext{
$\ddagger$ We are considering the bound states which have discrete spectrum. The quantity $q^{2}=M_{V}^{2}$ labels the degree of the radial excitation if we introduce it as $\chi=\chi\left(p^{2}, p \cdot q, q^{2}\right)$.
} 
where $C=i \gamma^{2} \gamma^{0}$, all the invariant amplitudes $\chi^{i}(u, x)$ are found to be even functions in $u: 13$

$$
\chi^{i}(u, x)=\chi^{i}(-u, x)
$$

\subsection{Homogeneous BS Equation}

The HBS equation reads

$$
T \chi=K \chi .
$$

The diagram for the HBS equation in the (improved) ladder approximation is shown
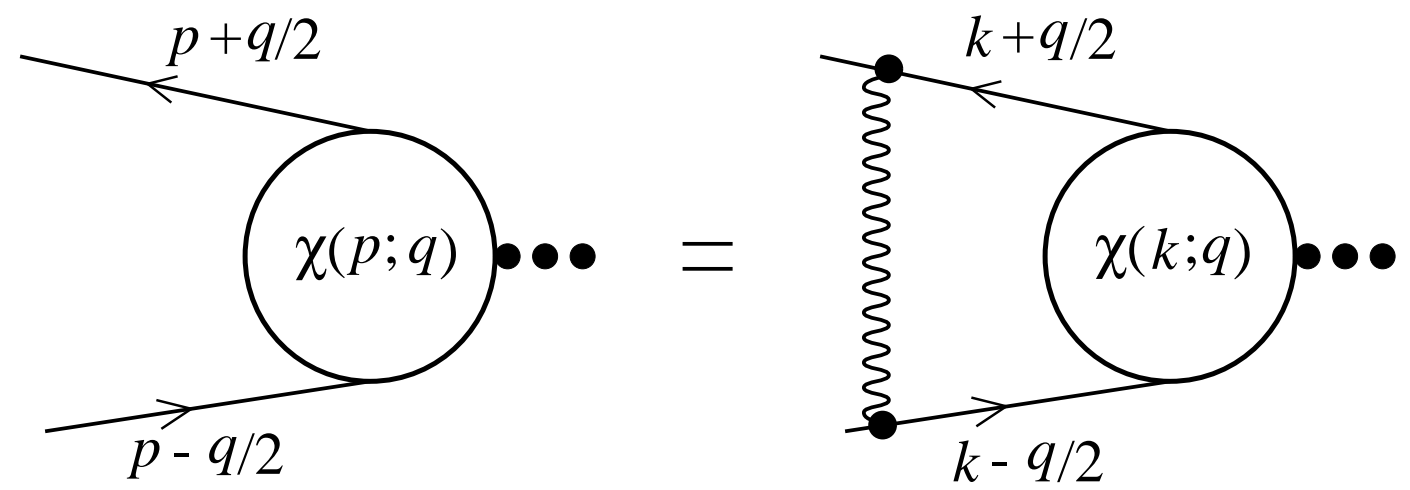

Figure 1: The Feynman diagram of the homogeneous BS equation (2.8) in the (improved) ladder approximation. The quark lines in the RHS of the equation are truncated.

in fig. 1. The kinetic part $T$ is given by

$$
T(p ; q)=S_{F}^{-1}(p+q / 2) \otimes S_{F}^{-1}(p-q / 2)
$$

with the tensor product notation

$$
(A \otimes B) \chi=A \chi B .
$$

In this approximation, the $\mathrm{BS}$ kernel $K$ is given by

$$
K(p, k)=C_{2} \frac{g^{2}(p, k)}{-(p-k)^{2}}\left(g_{\mu \nu}-\frac{(p-k)_{\mu}(p-k)_{\nu}}{(p-k)^{2}}\right) \gamma^{\mu} \otimes \gamma^{\nu}
$$

In eq. (2.8) we use the inner product rule

$$
K \chi(p ; q)=\int \frac{d^{4} k}{(2 \pi)^{4} i} K(p, k) \chi(k ; q) .
$$


In the improved ladder approximation we use the running coupling, the asymptotic form of which is given by

$$
\alpha\left(\mu^{2}\right) \equiv \frac{g^{2}\left(\mu^{2}\right)}{4 \pi} \underset{\mu \rightarrow \infty}{\longrightarrow} \frac{\alpha_{0}}{\ln \mu^{2}},
$$

where $\alpha_{0}=12 \pi /\left(11 N_{c}-2 N_{f}\right)$ with $N_{c}$ and $N_{f}$ being the number of colors and flavors, respectively.

The HBS equation (2.8) determines the eight invariant amplitudes $\chi^{1}, \cdots, \chi^{8}$ up to the overall normalization constant as well as the bound state mass $M_{V}$. Multiplying eq. (2.8) by the conjugate bispinor base $\bar{\Gamma}_{i}(p ; q, \epsilon) \equiv \gamma_{0} \Gamma_{i}\left(p^{*} ; q, \epsilon\right)^{\dagger} \gamma_{0}$, taking the trace of spinor indices and summing over the polarizations, we finally obtain

$$
T_{i j}(u, x) \chi^{j}(u, x)=\int \frac{y^{2} d y d v}{8 \pi^{3}} K_{i j}(u, x ; v, y) \chi^{j}(v, y)
$$

where the summation over the repeated indices is promised and

$$
\begin{aligned}
T_{i j}(u, x) & =\sum_{\epsilon} \frac{1}{4} \operatorname{tr}\left[\bar{\Gamma}_{i}(p ; q, \epsilon) T(p ; q) \Gamma_{j}(p ; q, \epsilon)\right] \\
K_{i j}(u, x ; v, y) & =\int_{-1}^{1} d \cos \theta \sum_{\epsilon} \frac{1}{4} \operatorname{tr}\left[\bar{\Gamma}_{i}(p ; q, \epsilon) K(p, k) \Gamma_{j}(k ; q, \epsilon)\right] .
\end{aligned}
$$

We omit the explicit forms of $T_{i j}(u, x)$ and $K_{i j}(u, x ; v, y)$ here because they are somewhat complicated. Due to the charge conjugation property (2.6) of the bispinor base, the $8 \times 8$ matrices $T_{i j}$ and $K_{i j}$ are real and self-conjugate:

$$
\begin{array}{lll}
T_{i j}(u, x)^{\dagger} & =T_{i j}(u, x)^{T} & =T_{j i}(u, x) \\
K_{i j}(u, x ; v, y)^{\dagger} & =K_{i j}(u, x ; v, y)^{T} & =K_{j i}(v, y ; u, x) .
\end{array}
$$

The complex conjugate and the transpose are taken in the $8 \times 8$ space. Using the property (2.7), we are allowed to restrict the integral region of $v$ to positive, $v>0$. The BS kernel in eq. (2.14) is replaced such that

$$
\int d v K_{i j}(u, x ; v, y) \chi^{j}(v, y)=\int_{v>0} d v\left[K_{i j}(u, x ; v, y)+K_{i j}(u, x ;-v, y)\right] \chi^{j}(v, y) .
$$

Thus, we can treat all the variables $u, x, v, y$ as positive values.

In order to solve eq. (2.14) numerically we discretize the momentum spaces $(u, x)$ and $(v, y)$. The effective parameterizations for the variables $u$ and $x$ are

$$
u=e^{U}, \quad x=e^{X},
$$


and similarly for $v$ and $y$. These new variables are discretized at $N_{B S}$ points evenly spaced in the intervals

$$
\begin{aligned}
& U, V \in\left[\lambda_{U}, \Lambda_{U}\right], \\
& X, Y \in\left[\lambda_{X}, \Lambda_{X}\right] .
\end{aligned}
$$

Then, the momentum integration becomes the summation

$$
\int_{v>0} y^{2} d y d v \cdots \quad D_{U} D_{X} \sum_{V, Y} V Y^{3} \cdots
$$

where

$$
\begin{aligned}
& D_{U}=\left(\Lambda_{U}-\lambda_{U}\right) /\left(N_{B S}-1\right), \\
& D_{X}=\left(\Lambda_{X}-\lambda_{X}\right) /\left(N_{B S}-1\right) .
\end{aligned}
$$

The BS kernel $K_{i j}(u, x ; v, y)$ has an integrable singularity at $(u, x)=(v, y)$ which comes from the pole in the propagator of the gauge boson (cf. eq. (2.11)). In order to avoid this singularity we regularize it by taking the four-point splitting 12

$$
\begin{aligned}
K_{i j}(u, x ; v, y) \rightarrow & \frac{1}{4}\left[K_{i j}\left(u, x ; v_{+}, y_{+}\right)+K_{i j}\left(u, x ; v_{+}, y_{-}\right)\right. \\
& \left.+K_{i j}\left(u, x ; v_{-}, y_{+}\right)+K_{i j}\left(u, x ; v_{-}, y_{-}\right)\right]
\end{aligned}
$$

where $v_{ \pm}=\exp \left(V \pm D_{U} / 4\right)$ and $y_{ \pm}=\exp \left(Y \pm D_{X} / 4\right)$.

Now, we are ready to solve eq. (2.14) numerically. The HBS equation (2.14) becomes a finite dimensional linear equation in terms of the invariant BS amplitude $\chi^{i}$. All we have to do is to obtain simultaneously the bound state mass $M_{V}$ and the corresponding BS amplitude $\chi$.

\subsection{Normalization Condition}

First, we introduce the conjugate $\mathrm{BS}$ amplitude $\bar{\chi}(p ; q, \epsilon)$ as

$$
\langle V(q, \epsilon)|\mathrm{T} \psi(y) \bar{\psi}(x)| 0\rangle=-e^{i q X} \int \frac{d^{4} p}{(2 \pi)^{4}} e^{i p r} \bar{\chi}(p ; q, \epsilon) .
$$

The relation between the conjugate BS amplitude and the BS amplitude is given by

$$
\bar{\chi}(p ; q, \epsilon)=\gamma_{0}\left[\chi\left(p^{*} ; q, \epsilon\right)\right]^{\dagger} \gamma_{0}
$$

Now, let us fix the normalization of the BS amplitude $\chi$. Usually the bound state $|V(q, \epsilon)\rangle$ is normalized by

$$
\left\langle V\left(q^{\prime}, \epsilon^{\prime}\right) \mid V(q, \epsilon)\right\rangle=2 q_{0}(2 \pi)^{3} \delta_{\epsilon, \epsilon^{\prime}} \delta^{3}\left(\boldsymbol{q}-\boldsymbol{q}^{\prime}\right) .
$$


This condition fixes the normalization of $\chi$ via its definition (2.1). The convenient form for the normalization of $\chi$ is given by the Mandelstam formula

$$
\left\langle\chi\left|\frac{\partial T}{\partial q^{\mu}}\right| \chi\right\rangle=2 q_{\mu} .
$$

Here we use the bra-ket notation [6]

$$
\langle\chi|A| \psi\rangle=\int \frac{d^{4} p}{(2 \pi)^{4} i} \operatorname{tr}[\bar{\chi}(p ; q)(A \psi)(p ; q)]
$$

with the trace taken over the spinor indices and all the other inner degrees of freedom. Multiplying eq. (2.26) by $q^{\mu}$ and using $q^{\mu}\left(\partial / \partial q^{\mu}\right)=M_{V}\left(\partial / \partial M_{V}\right)$ we get

$$
2 M_{V}=\left\langle\chi\left|\frac{\partial T}{\partial M_{V}}\right| \chi\right\rangle
$$

It is easy to find the normalization condition in component form as

$$
2 M_{V}=\frac{N_{c}}{2 \pi^{3}} \int x^{2} d x d u\left(\chi^{i}(-u, x)\right)^{*} \frac{\partial T_{i j}}{\partial M_{V}} \chi^{j}(u, x)
$$

\section{The Method for Solving the Homogeneous Bethe-Salpeter Equation}

In this section we study the method for solving the homogeneous Bethe-Salpeter (HBS) equation and explain how to apply it to the numerical calculation.

Let us start with writing down the HBS equation (2.8):

$$
T \chi=K \chi
$$

This HBS equation simultaneously determines the mass spectrum $M_{V}^{(1)}, M_{V}^{(2)}, M_{V}^{(3)}$, $\cdots$ of the bound states and the BS amplitudes $\chi_{1}(p ; q, \epsilon), \chi_{2}(p ; q, \epsilon), \chi_{3}(p ; q, \epsilon), \cdots$ up to their normalizations. Therefore, to solve the HBS equation is to find a mass $M_{V}$ of a certain bound state and its corresponding BS amplitude $\chi(p ; q, \epsilon)$. However, it is difficult to solve HBS equation (3.1) as it is. For definiteness, we choose the suffix $n$ as $M_{V}^{(1)} \leq M_{V}^{(2)} \leq M_{V}^{(3)} \leq \cdots$.

Usually, the coupling constant $\alpha$ is regarded as an eigenvalue of HBS equation (3.1) for a given bound state mass, [3] where we use the ladder and the constant mass approximations simultaneously. The dependence on the coupling constant is isolated in the BS kernel and the coupling factors out. As far as we adopt those approximations, this is extended to the running coupling case by regarding the overall 
constant $\alpha_{0}$ given in eq. (2.13) as an eigenvalue. On the contrary, if we consistently use the full propagator determined by the SD equation in the same approximation as that for the BS equation, the kinetic part $T$ also depends on the coupling $\alpha$ as well as the BS kernel $K$ does. Then, the HBS equation (3.1) cannot be regarded as a simple linear eigenvalue equation for the coupling.

The original idea to regard the HBS equation as a linear eigenvalue equation for the coupling and the BS amplitude is easily extended to generic cases. By introducing a 'fictitious' eigenvalue $\lambda$ we interpret the HBS equation (3.1) as a linear eigenvalue equation for a given bound state mass $M_{V}$ :

$$
\left(\frac{1}{\lambda}\right) T \chi=K \chi
$$

where the kinetic part $T$ is regarded as a weight function. We calculate the bound state mass for a given coupling $\alpha$ numerically from this equation. When we consider the mass $M_{V}$ as an input parameter, the form of eq. (3.2) guarantees the solvability: we can solve eq. (3.2) by standard linear algebraic techniques for eigenvalue equations, and obtain the solutions of the eigenvalue $\lambda$. The fictitious eigenvalue is essentially the same as the coupling constant, $\lambda \propto \alpha$, when we use both the (improved) ladder and constant fermion mass approximations. The resultant HBS equation (3.2) simultaneously describes various systems parameterized by the coupling constant $\alpha$.

In what follows we first study the basic notions of the eigenvalue equation (3.2) and then we explain the method for solving the original HBS equation (3.1). We note that we have to check the existence of the discrete spectrum of $\lambda$. As we will show in the next section, there exists the discrete spectrum in the ladder and constant mass approximations. Here and henceforth we consider such case. In order to distinguish the quantum numbers of the eigenvalue equation (3.2) from that of the original HBS equation (3.1) we attach primes to them like $n^{\prime}$ or $1^{\prime}, 2^{\prime}, 3^{\prime}, \cdots$ when we solve eq. (3.2) to obtain the spectrum of $\lambda$ for a given $M_{V}$. The obtained eigenvalue $\lambda$ is a function of the bound state mass parameter $M_{V}$, so we write the dependence explicitly as $\lambda_{n^{\prime}}\left(M_{V}\right)$. For the convenience of the numerical calculation we define the numbering of $\left\{\lambda_{n^{\prime}}\left(M_{V}\right)\right\}$ so as to satisfy

$$
0 \leq \lambda_{1^{\prime}}\left(M_{V}\right) \leq \lambda_{2^{\prime}}\left(M_{V}\right) \leq \lambda_{3^{\prime}}\left(M_{V}\right) \leq \cdots,
$$

where the equality holds when the eigenvalues are degenerate. In our numerical calculation we encounter the accidental degeneracy of the two eigenvalues at some point $M_{V}$. We call this phenomena 'level crossing'.

There are two kinds of norms of the BS amplitude in eq. (3.2). The quantity $\left\langle\chi_{n^{\prime}}\left|\partial T / \partial M_{V}\right| \chi_{n^{\prime}}\right\rangle$, which we call $T^{\prime}$-norm, gives the normalization for the BS am- 
plitude as in eq. (2.28). The non-positive $T^{\prime}$-norm states are ghosts and cannot be normalized. So, we consider only the states which have positive $T^{\prime}$-norm. The quantity $\left\langle\chi_{n^{\prime}}|(-T)| \chi_{n^{\prime}}\right\rangle$, which we call $T$-norm, gives also a natural norm for the eigenvalue equation (3.2) in the following sense. In the case where the eigenvalue $\lambda$ is not always real, it is easy to derive the well-known relation in linear algebra:

$$
\left(1 / \lambda_{n^{\prime}}-1 / \lambda_{m^{\prime}}^{*}\right)\left\langle\chi_{m^{\prime}}|(-T)| \chi_{n^{\prime}}\right\rangle=0
$$

This relation says that the eigenvalue $\lambda$ is real otherwise the corresponding state $\chi$ has zero $T$-norm, and the state $\left|\chi_{n^{\prime}}\right\rangle \mid$ can form a complete orthonormal set under the inner product $(\chi, \psi) \equiv\langle\chi|(-T)| \psi\rangle$.

For the purpose that we find the solutions with $\lambda=1$, it is enough to consider $\lambda$ is real and positive. [We do not consider the possibility that $\lambda$ becomes negative, because it implies the repulsive interaction which cannot form any bound states.] Then, we throw away all non-real eigenvalues and its corresponding eigenvectors. Further, we do not consider the complex $M_{V}$ in solving eq. (3.2). If $M_{V}$ is complex, the corresponding state is complex ghost and is physically unacceptable.

Now, let us consider the relation between the linear eigenvalue equation (3.2) and the original HBS equation (3.1). What we obtain from (3.2) is the set $\left\{\lambda_{n^{\prime}}, \chi_{n^{\prime}}\right\}$ for a given $M_{V}$, but what we have to find out is the set $\left\{M_{V}^{(n)}, \chi_{n}\right\}$. If we find an eigenvalue $\lambda_{n^{\prime}}$ and its corresponding eigenvector $\chi_{n^{\prime}}$ such that $\lambda_{n^{\prime}}=1$ for a certain value of the mass $M_{V}$, this solution also satisfies the original equation (3.1):

$$
0=\left(T-\lambda_{n^{\prime}} K\right) \chi_{n^{\prime}}=(T-K) \chi_{n^{\prime}}
$$

Namely, the set $\left\{M_{V}, \chi_{n^{\prime}} \mid \lambda_{n^{\prime}}=1\right\}$ is nothing but a solution of the HBS equation (3.1). Of course, the other states $m^{\prime} \neq n^{\prime}$ which do not satisfy $\lambda_{m^{\prime}}=1$ are out of the case. Then, the physical spectrum $\left\{M_{V}^{(n)} \mid n \in \mathbf{N}\right\}$ of the bound state masses is determined by the following set of intrinsic relations

$$
\lambda_{1^{\prime}}\left(M_{V}\right)=1, \lambda_{2^{\prime}}\left(M_{V}\right)=1, \lambda_{3^{\prime}}\left(M_{V}\right)=1, \cdots .
$$

Clearly, all the roots of these equations give the solutions of the original HBS equation (3.1).

Next, we explain how to input the mass $M_{V}$ in eq. (3.2) by a systematic manner for tuning the eigenvalue to unity $\left(\lambda_{n^{\prime}}=1\right)$ numerically. For this purpose we first consider the differentiability of the eigenvalue $\lambda\left(M_{V}\right)$. In general the function $\lambda_{n^{\prime}}\left(M_{V}\right)$ defined by eq. (3.3) is not always differentiable at level crossing point. We modify the definition of the numbering $\left(\lambda_{n^{\prime}} \rightarrow \lambda_{n}\right)$ so that the functional forms of the eigenvalues 
become always differentiable. In order to make differentiable form $\lambda\left(M_{V}\right)$ for all range of $M_{V}$ we invoke the eigenvalue equation (3.2) itself. Differentiating eq. (3.2) with respect to $M_{V}$, we have[3]

$$
\frac{d \lambda\left(M_{V}\right)}{d M_{V}}=-\lambda\left(M_{V}\right) \frac{\left\langle\chi\left|\frac{\partial T}{\partial M_{V}}\right| \chi\right\rangle}{\langle\chi|(-T)| \chi\rangle},
$$

where we use the HBS equation for the conjugate BS amplitude. For normalizable BS amplitudes we put $\left\langle\chi\left|\partial T / \partial M_{V}\right| \chi\right\rangle=2 M_{V}$ according to eq. (2.28). The equation (3.7) implies that if the BS amplitude $\chi$ is normalizable and have non-vanishing $T$ norm, the derivative of the corresponding eigenvalue $\lambda$ exists. Let $\lambda_{n}\left(M_{V}\right)(n \in \mathbf{N})$ denote one of such eigenvalues and let $\chi_{n}$ denote its corresponding eigenvector. Once we define the numbering (or we can say tagging) $\lambda_{n}\left(M_{V}\right)$ at a suitable point $M_{V}$, we can uniquely identify the functional form of $\lambda_{n}\left(M_{V}\right)$ according to the "differential equation" (3.7). In other words, the differential equation (3.7) with a suitable initial condition uniquely determines the form of $\lambda_{n}\left(M_{V}\right)$.

Here, we explain the systematic method to tune the eigenvalue. We first consider the case that the level crossing does not occur, and we identify $\lambda_{n}\left(M_{V}\right)=\lambda_{n^{\prime}}\left(M_{V}\right)$ for $n=1,2, \cdots$. The relation (3.7) tells us the response of the eigenvalue $\lambda_{n^{\prime}}\left(M_{V}\right)$ for the bound state mass $M_{V}$. If we update the bound state mass to $M_{V}+\delta M_{V}$, then the eigenvalue $\lambda_{n^{\prime}}$ changes to $\lambda_{n^{\prime}}+\delta M_{V}\left(d \lambda_{n^{\prime}} / d M_{V}\right)$ according to the relation (3.7). In order to find the root of the equation $\lambda_{n^{\prime}}\left(M_{V}\right)=1$ numerically, we are better to use the Newton's method. The bound state mass is updated according to

$$
\begin{aligned}
M_{V} & \rightarrow M_{V}+\delta M_{V}, \\
\delta M_{V} & =\frac{\left\langle\chi_{n^{\prime}}|(-T)| \chi_{n^{\prime}}\right\rangle}{\left\langle\chi_{n^{\prime}}\left|\frac{\partial T}{\partial M_{V}}\right| \chi_{n^{\prime}}\right\rangle}\left(1-\frac{1}{\lambda_{n^{\prime}}}\right) .
\end{aligned}
$$

When $\lambda_{n^{\prime}}\left(M_{V}\right)$ is not differentiable at a level crossing point $M_{0}, \lambda_{n^{\prime}}$ and $\chi_{n^{\prime}}$ in eq. (3.8) should be replaced with $\lambda_{n}$ and $\chi_{n}$ respectively. The derivative $d \lambda_{n^{\prime}} / d M_{V}$ has two different values at $M_{0}$ depending on how to take the limits, $M_{V} \searrow M_{0}$ or $M_{V} \nearrow M_{0}$. If the difference between these two values is not so large, it is not a problem for the purpose of updating $\lambda_{n^{\prime}}$ : we do not need the precise value of $M_{V}$ so as to satisfy $\lambda_{n^{\prime}}\left(M_{V}\right)=1$ at one time, whereas we need only more suitable value of $M_{V}$ than the previous trial value of $M_{V}$. Therefore, we generally use the updating procedure (3.8).

It is important to study the property of the spectrum of $\lambda$ in order to know which of the states the solution $\left\{M_{V}, \chi_{n^{\prime}} \mid \lambda_{n^{\prime}}=1\right\}$ is corresponding to, the ground state or the the first excited state or the other states. In this section we assume that the solutions have positive $T$-norm below. [Indeed, our numerical calculations given in 
the next section satisfies this assumption.] Under this assumption, all eigenvalues $\lambda_{n}\left(M_{V}\right)(n=1,2, \cdots)$ in consideration are differentiable and decreasing functions. Thus, all $\lambda_{n^{\prime}}\left(M_{V}\right)$ 's are also decreasing functions, and each equation $\lambda_{n^{\prime}}\left(M_{V}\right)=1$ in eq. (3.6) has only one root at most. Many models suggest that $\lambda\left(M_{V}\right)$ is a decreasing function (see for example, ref. [3]). [Smaller value of $M_{V}$ means larger binding energy which should be realized by larger coupling to form tight bound state. $\lambda$ is roughly the same as the coupling.]

We notice that there is a possibility that some of the equations in (3.6) have no solution, e.g., $\lambda_{n^{\prime}}\left(M_{V}\right) \neq 1$, for any real input value $M_{V}$. Those $\lambda^{\prime}$ 's which are already smaller than unity $(\lambda<1)$ at $M_{V}=0$ never have the solution of $\lambda\left(M_{V}\right)=1$. In this case the eigenvalue and its corresponding state $\left(\lambda_{n^{\prime}}, \chi_{n^{\prime}}\right)$ has no physical correspondence. 3 In other word, the state $\chi_{n^{\prime}}$ is annihilated in the physical states which are determined in terms of eq. (3.6). We call this phenomena the "level annihilation". We actually encounter the level annihilation in the running coupling case, which is studied in the next section.

Now, we will establish the identifications of the physical states $\left\{M_{V}^{(n)}, \chi_{n}\right\}$ with the solutions $\left\{M_{V}, \chi_{m^{\prime}} \mid \lambda_{m^{\prime}}\left(M_{V}\right)=1\right\}$. In the numerical calculation these identifications are easy tasks. We calculate the set of eigenvalues $\left\{\lambda_{n^{\prime}}\right\}$ from $M_{V}=0$ to a certain large value. When we use the constant mass and (improved) ladder approximation, the point $M_{V}=2 \mathrm{~m}$ is the threshold for the constituent fermions to be liberated from the bound state. So, it is enough to calculate $\lambda$ up to $M_{V}=2 m$. Supposing that there are $l-1$ level annihilations, we obtain $\lambda_{1^{\prime}} \leq \cdots \leq \lambda_{(l-1)^{\prime}}<1 \leq \lambda_{l^{\prime}} \leq \cdots$ at $M_{V}=0$. Then, the l-th eigenvalue $\lambda_{l^{\prime}}$, which becomes unity first as increasing the input parameter $M_{V}$ and its corresponding $B S$ amplitude $\chi_{l^{\prime}}$ give the ground state. The $(l+1)$-th eigenvalue $\lambda_{(l+1)}$ which becomes unity second and its corresponding $B S$ amplitude $\chi_{m^{\prime}}$ give the first excited state, and so on.

Finally, we consider the identification of $\lambda_{n}\left(M_{V}\right)$ with $\lambda_{m^{\prime}}\left(M_{V}\right)$ under the level crossing. When there is no degeneracy between any two eigenvalues of $\lambda_{m^{\prime}}$ 's, we can uniquely identify the form of $\lambda_{n}\left(M_{V}\right)$ according to the continuity: $\lambda_{n}\left(M_{V}\right)=\lambda_{n^{\prime}}\left(M_{V}\right)$ for ${ }^{\forall} n \in \mathbf{N}$. [In a model a curve of $\lambda\left(M_{V}\right)$ terminates at some point $M_{V}$ [24], but the continuity of the curve does not spoil.] However, in general cases there may occur the level crossing. The level crossing is that the accidental degeneracy of states for a specific value of the bound state mass $M_{V}$. There exist two possible kinds of level crossing: 1) $\lambda_{n^{\prime}}$ and $\lambda_{m^{\prime}}$ are degenerate while both are still differentiable; 2) $\lambda_{n^{\prime}}$

$\S$ If we allow $M_{V}$ to take complex value, we may always have roots of eq. (3.6). But, in our calculation we do not consider complex bound state mass because it is unphysical. 
and $\lambda_{m^{\prime}}$ are not differentiable at level crossing point. Below, we discuss the latter kind of level crossing. The important thing here is that $\lambda_{n}\left(M_{V}\right)$ is defined by the differentiability (3.7) while $\lambda_{n^{\prime}}\left(M_{V}\right)$ is defined by the ordering (3.3). For simplicity here we consider the case when only two states become degenerate at one time with different slopes of $\lambda\left(M_{V}\right)$. If one level crossing between the first and the second smallest $\lambda$ 's occurs at $M_{V}=M_{V 1}$ then $\lambda_{n}\left(M_{V}\right)=\lambda_{n^{\prime}}\left(M_{V}\right)(n=1,2,3, \cdots)$ for $M_{V}>M_{V 1}$ and $\lambda_{2}\left(M_{V}\right)=\lambda_{1^{\prime}}\left(M_{V}\right), \lambda_{1}\left(M_{V}\right)=\lambda_{2^{\prime}}\left(M_{V}\right)$ and $\lambda_{n}\left(M_{V}\right)=\lambda_{n^{\prime}}\left(M_{V}\right)$ $(n=3,4, \cdots)$ for $M_{V}<M_{V 1}$. This identification is easily understood in the general cases, where the differentiability of $\lambda_{n}\left(M_{V}\right)$ uniquely make us identify its form. As a result, the phenomena of the level crossing is that two differentiable curves $\lambda_{m}\left(M_{V}\right)$ and $\lambda_{n}\left(M_{V}\right)$ cross at a point $M_{V}=M_{V \text { cross }}$.

\section{Numerical Calculation}

In this section, we first study the functional form of $\lambda\left(M_{V}\right)$ in our numerical method. We consider the running coupling case (improved ladder approximation) in the constant fermion mass approximation. Next, we evaluate the bound state mass determined by the original HBS equation eq. (3.1). This study is motivated by the $\bar{q} q$ quarkonia in QCD. Here and henceforth, we rescale all dimensionful quantities by $\Lambda_{\mathrm{QCD}}$ otherwise stated. $\Lambda_{\mathrm{QCD}}$ is defined by the blow-up scale of the one-loop running coupling in the improved ladder model.

Let us consider the important things for the numerical calculations. As discussed before, in the improved ladder approximation the asymptotic form of the running coupling is given by the one-loop renormalization group equation, but we have no idea for the functional form in the low energy region. As in ref. [13] we use the form of the running coupling:

$$
\alpha\left(\mu^{2}\right) \equiv \frac{g^{2}\left(\mu^{2}\right)}{4 \pi}=\alpha_{0} \times \begin{cases}\frac{1}{t} & \text { if } t_{F}<t \\ \frac{1}{t_{F}}+\frac{\left(t_{F}-t_{C}\right)^{2}-\left(t-t_{C}\right)^{2}}{2 t_{F}^{2}\left(t_{F}-t_{C}\right)} & \text { if } t_{C}<t<t_{F} \\ \frac{1}{t_{F}}+\frac{\left(t_{F}-t_{C}\right)}{2 t_{F}^{2}} & \text { if } t<t_{C}\end{cases}
$$

where $t=\ln \mu^{2}$ and $\alpha_{0}=12 \pi /\left(11 N_{c}-2 N_{f}\right)$ with $N_{c}$ and $N_{f}$ being the number of colors and flavors, respectively. In this paper we fix $N_{c}=N_{f}=3$ and $t_{F}=0.5$ and $t_{C}=2.0$.

In the numerical calculation we have to take care of covering the supports of both $\langle\chi|(-T)| \chi\rangle\left(T\right.$-norm) and $\left\langle\chi\left|\partial T / \partial M_{V}\right| \chi\right\rangle\left(T^{\prime}\right.$-norm). Two supports of the norms 
should lie within the momentum cutoffs $\left[\lambda_{U}, \Lambda_{U}\right]$ and $\left[\lambda_{X}, \Lambda_{X}\right]$ given in eq. (2.19), otherwise we fail to obtain the precise values of the eigenvalue $\lambda$ and its corresponding BS amplitudes as well as the bound state masses. Covering the support of $T$-norm is necessary for finding out the correct value of the fictitious eigenvalue $\lambda$ by our updating method given in sect. [3. The $T^{\prime}$-norm plays the role for determining whether the state is a physical state $\left(T^{\prime}>0\right)$ or a ghost state $\left(T^{\prime} \leq 0\right)$. The ratio of $T^{\prime}$ - and $T$-norms is essential for checking the error $\delta M_{V}$ defined in eq. (3.8) to converge the correct value of the bound state mass.

For the evaluation of $M_{V}^{(1)}$, we need to cover the support of $\left\langle\chi_{1^{\prime}}|(-T)| \chi_{1^{\prime}}\right\rangle$ if there is no level annihilation. When we evaluate $M_{V}^{(2)}$ we need to cover both the supports of $\left\langle\chi_{1^{\prime}}|(-T)| \chi_{1^{\prime}}\right\rangle$ and $\left\langle\chi_{2^{\prime}}|(-T)| \chi_{2^{\prime}}\right\rangle$, and so on. This is because the excited state $\chi_{n^{\prime}}$ should be orthogonal to the lower states $\chi_{1^{\prime}}, \chi_{2^{\prime}}, \cdots, \chi_{(n-1)^{\prime}}$ in the sense of eq. (3.4). (7) In the following calculations we carefully cover the $T$-norm and $T^{\prime}$-norm supports of the first two states, i.e., $\chi_{1^{\prime}}$ and $\chi_{2^{\prime}}$. We show the typical example of the supports of $T$-norm and $T^{\prime}$-norm for the choice $m=1.0$ and $M_{V}=1.2 \mathrm{in} \mathrm{fig.} \mathrm{2.} \mathrm{[The} \mathrm{condition}$

(a)

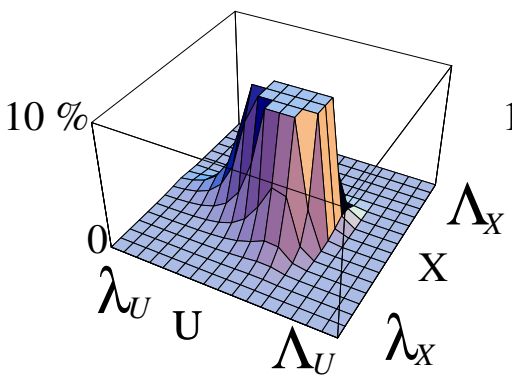

(b)

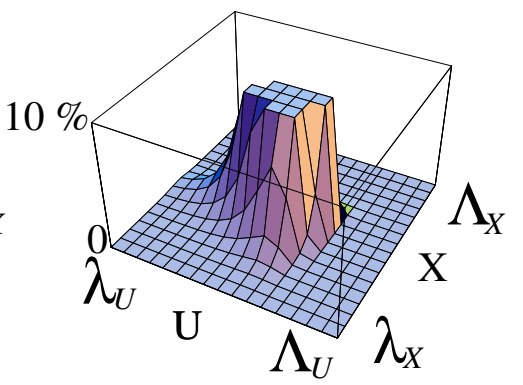

Figure 2: The supports of a) $T$-norm and b) $T^{\prime}$-norm for the choice $m=1.0$ and $M_{V}=1.2$ with $N_{B S}=17$ for the state $\chi_{1^{\prime}}$. The upper $9 / 10$ of each figures is clipped. Horizontal axes of $U$ and $X$ are parameterized by eq. (2.18). Summing up all values on the lattice points give us the norm for a) and b).

$\lambda_{1^{\prime}}\left(M_{V}\right)=1$ is satisfied by this parameter choice.]

\footnotetext{
I If we calculate the decay constant, we also need to cover the support of $T^{\prime}$-norm in a similar reason.
} 


\subsection{The spectrum of $\lambda$}

First, we show the functional form of the fictitious eigenvalue $\lambda_{n^{\prime}}\left(M_{V}\right)$ with a choice $m=1.0$ in fig. 3. This figure obviously shows that the smallest three $\lambda_{n^{\prime}}\left(M_{V}\right)$ 's

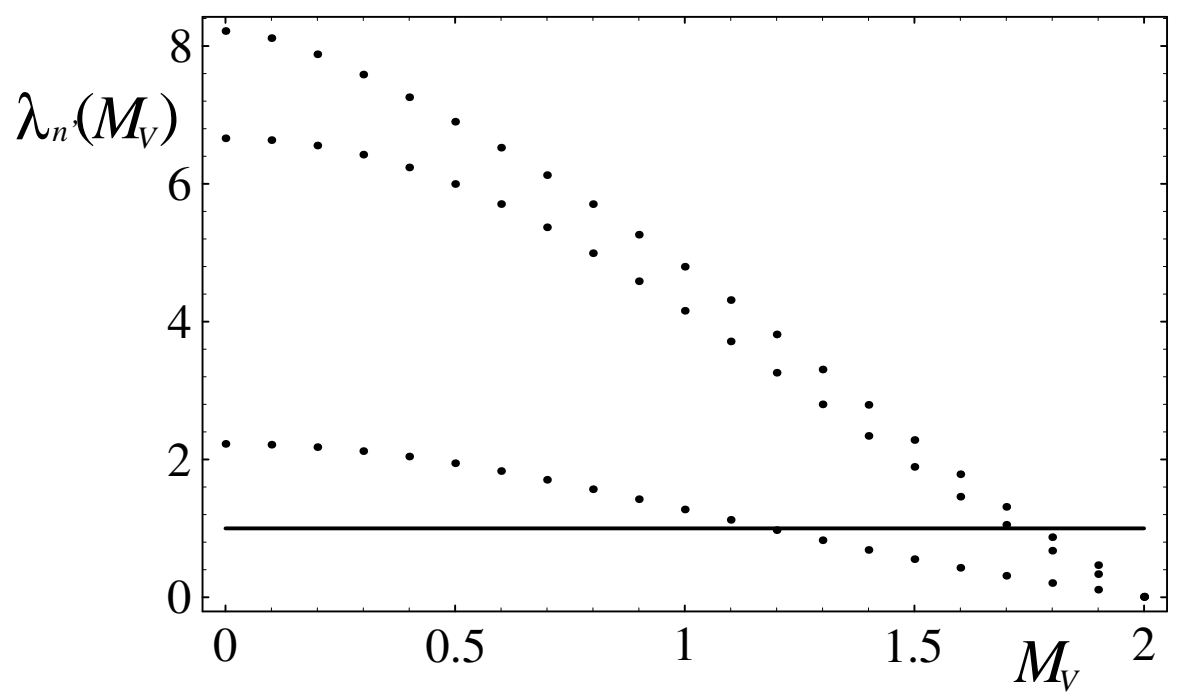

Figure 3: The functional forms of the smallest three $\lambda_{n^{\prime}}\left(M_{V}\right)$. The horizontal line indicates $\lambda_{n^{\prime}}\left(M_{V}\right)=1$. We use $N_{B S}=17$.

are monotonically decreasing functions and there are no level crossing and level annihilation. The eigenvalues $\lambda_{1^{\prime}}\left(M_{V}\right)$ and $\lambda_{2^{\prime}}\left(M_{V}\right)$ are the first and the second to become unity respectively when the bound state mass increases from zero. Then, the states $\chi_{1^{\prime}}$ and $\chi_{2^{\prime}}$ are corresponding to the ground state and the first excited state, respectively. At the free fermion threshold $M_{V}=2 m$ all eigenvalues of $\lambda$ become zero. There are free-state solutions which satisfy $T \chi=0$ (eq. (3.2) with $\lambda=0$ ). At $M_{V}=0$ all three slopes of $\lambda_{n^{\prime}}\left(M_{V}\right)$ become flat smoothly, i.e., $d \lambda_{n^{\prime}}\left(M_{V}\right) / d M_{V} \rightarrow 0$ as $M_{V} \rightarrow 0$. As in eq. (3.7), this means that $T^{\prime}$-norm vanishes $\left\langle\chi_{n^{\prime}}\left|\partial T / \partial M_{V}\right| \chi_{n^{\prime}}\right\rangle=0$ consistently with the normalization condition (2.28). Actually, the calculated ratio of $T^{\prime}$-norm over $T$-norm vanishes at $M_{V}=0$. This result implies that the discrete spectrum at $M_{V}=0$ is the set of normal states in the sense of the limit $M_{V} \rightarrow 0$.

Second, we study the cases for smaller fermion masses. We show the spectrum of the smallest three $\lambda_{n^{\prime}}\left(M_{V}\right)$ with $m=0.5$ and 0.2 in fig. 4 . When we chose $m=0.5$, the smallest eigenvalue $\lambda_{1^{\prime}}\left(M_{V}\right)$ takes the maximum value unity at the vanishing bound state mass $M_{V}=0$ as in fig. $\Psi$ (a). This means that $m=0.5$ is a critical value for the level annihilation. If we chose smaller value of the fermion mass $(m<0.5)$, then the level annihilation of the state $\chi_{1^{\prime}}$ will occur. Accordingly, the mass of the 
ground state changes discretely from zero to $M_{V}=0.73$ around $m \sim 0.5$. When we chose $m=0.2$ as in fig. 4 (b), the smallest eigenvalue $\lambda_{1^{\prime}}\left(M_{V}\right)$ is always smaller than unity and never reaches to unity over the range $0 \leq M_{V} \leq 2 \mathrm{~m}$. In other words, the equation $\lambda_{1^{\prime}}\left(M_{V}\right)=1$ has no solution for $0 \leq M_{V} \leq 2 m$. This means that the state $\chi_{1^{\prime}}$ has no correspondence with the physical solutions of eq. (3.1), i.e., the level annihilation occurs. Then, $\chi_{2^{\prime}}, \chi_{3^{\prime}}, \cdots$ are corresponding to the ground state, first excited state and so on, respectively. If the constant fermion mass becomes much smaller than 0.2 , the next smallest eigenvalue $\lambda_{2^{\prime}}\left(M_{V}\right)$ will become always smaller than unity as well as $\lambda_{1^{\prime}}\left(M_{V}\right)$. When $m=0$, maximal number of states will fail to have any physical correspondences.

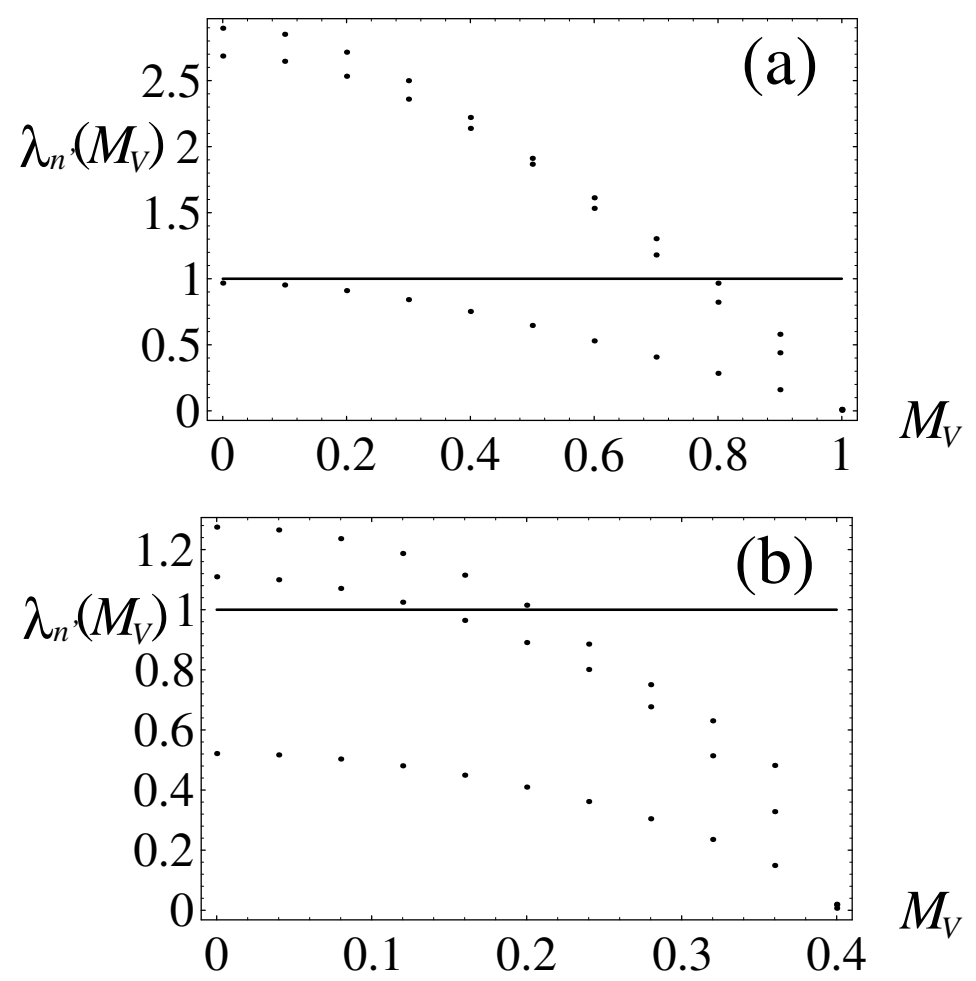

Figure 4: The plots of the smallest three $\lambda_{n^{\prime}}\left(M_{V}\right)$. We use constant fermion masses $m=0.5$ in fig. (a) and $m=0.2$ in fig. (b). The horizontal lines indicate $\lambda_{n^{\prime}}\left(M_{V}\right)=1$. We use $N_{B S}=11$.

Here it is important to notice that figs. 3 and 4 show that the slopes of the smallest three $\lambda_{n^{\prime}}\left(M_{V}\right)$ are negative. Further, we actually observe that all states in consideration have positive $T$ - and $T^{\prime}$-norms, and this leads, by eq. (3.7), to the fact that the corresponding $\lambda_{n^{\prime}}\left(M_{V}\right)$ (or $\lambda_{n}\left(M_{V}\right)$ ) are decreasing functions. This result 
supports our assumption that all normal solutions have positive $T$-norm, then all eigenvalues $\lambda_{n^{\prime}}\left(M_{V}\right)$ are decreasing functions.

Let us reconsider the level annihilation from the other side. Since the largest value of $\lambda_{n^{\prime}}\left(M_{V}\right)$ is realized at the vanishing bound state mass $M_{V}=0$, it is enough to calculate the eigenvalues $\lambda_{1^{\prime}}, \lambda_{2^{\prime}}, \cdots$ at $M_{V}=0$ for various constant fermion masses in order to see whether the level annihilation occurs. We show the result in fig. 5.

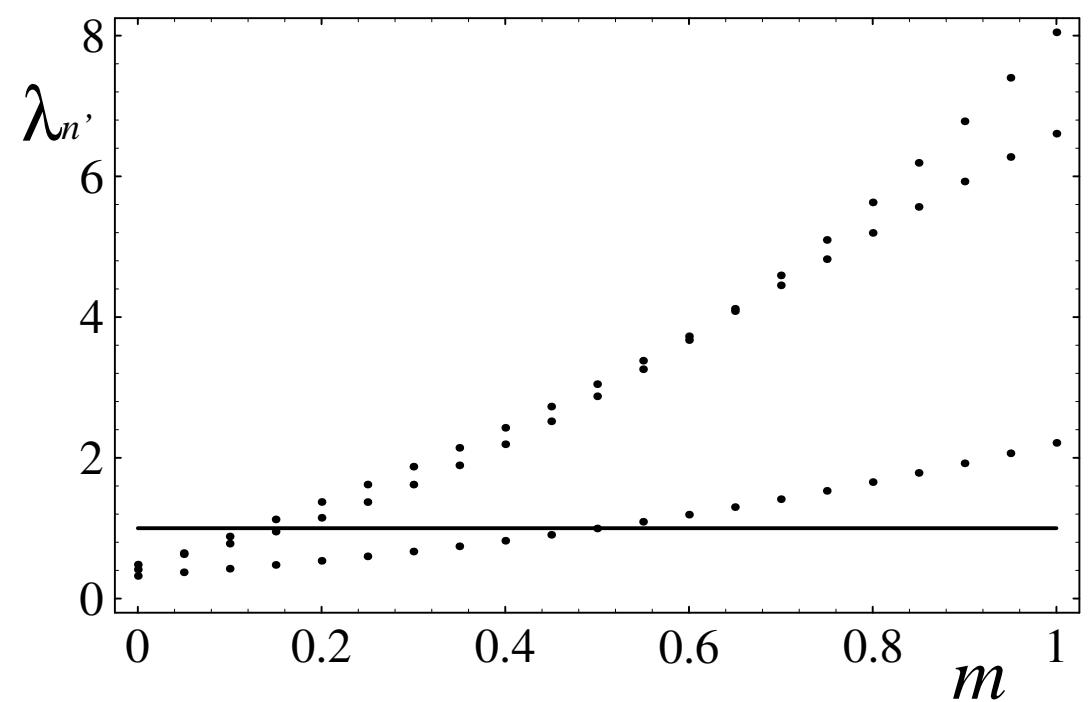

Figure 5: The smallest three $\lambda_{n^{\prime}}$ against $m$ at the vanishing bound state mass $M_{V}=0$. We use $N_{B S}=14$. At each value of the fermion mass $m$ we distinguish the three points by the definition of its ordering $\lambda_{1^{\prime}} \leq \lambda_{2^{\prime}} \leq \lambda_{3^{\prime}}$

This figure shows that the first level $\chi_{1^{\prime}}$ is annihilated at $m=0.5$, the second level $\chi_{2^{\prime}}$ are annihilated at $m=0.16$ and the third level $\chi_{3^{\prime}}$ at $m=0.13$ and so on. In other words, the ground state mass is given as a solution of $\lambda_{1^{\prime}}\left(M_{V}\right)=1$ for $m>0.5$, while we have to solve $\lambda_{2^{\prime}}\left(M_{V}\right)=1$ for $0.16<m<0.5$. [The two level crossings with respect to the fermion mass seem to occur around $m=0.05$ and 0.64.] Recall that we are considering only real value of $M_{V}$. If we allowed the bound state mass $M_{V}$ to take complex value, then we could always find the solution of $\lambda_{n^{\prime}}\left(M_{V}\right)=1$, and might have no level annihilation. Since we are interested in physical states, this solution is beyond the scope of this paper.

Next, let us consider the existence of the discrete spectrum, which we assume so far for simplicity. It is sufficient to study at the vanishing bound state mass $M_{V}=0$. As is seen from eq. (4.1), the running coupling becomes constant in the low energy 
region, which means the low energy behavior of the model is similar to that of the strong coupling QED. For a type of BS equation in the fixed coupling case, there is no discrete spectrum of $\lambda$ (or $\alpha_{0} \lambda$ ) for the tightly bound state, $M_{V}=0$ [20. So, it is important to investigate whether the discrete spectrum exists in the running coupling case. Figure 5 shows that the there exists discrete spectrum for all range of the fermion mass $m>0$. Clearly the state $\chi_{1^{\prime}}$ is one of the discrete states: if we had continuous states, the differences $\lambda_{2^{\prime}}-\lambda_{1^{\prime}}$ and $\lambda_{3^{\prime}}-\lambda_{1^{\prime}}$ would be of order $O\left(1 / N_{B S}\right)$. But our result shows the order-one differences. To confirm the existence of the discrete spectrum of the fictitious eigenvalue $\lambda$, we use the three choices of lattice sizes $N_{B S}=11,14$ and 17. We show the values of $\lambda_{1^{\prime}}, \lambda_{2^{\prime}}$ and $\lambda_{3^{\prime}}$ for the fermion mass $m=1.0$ in table 1. Each $\lambda_{n^{\prime}}$ changes its value by $O\left(1 / N_{B S}\right)$ as the

\begin{tabular}{|c||c|c|c|}
\hline$N_{B S}$ & 11 & 14 & 17 \\
\hline$\lambda_{1^{\prime}}$ & 2.24 & 2.21 & 2.23 \\
$\lambda_{2^{\prime}}$ & 6.42 & 6.61 & 6.66 \\
$\lambda_{3^{\prime}}$ & 7.85 & 8.05 & 8.22 \\
\hline
\end{tabular}

Table 1: The dependences of the fictitious eigenvalues $\lambda_{1^{\prime}}, \lambda_{2^{\prime}}$ and $\lambda_{3^{\prime}}$ on the lattice size for $M_{V}=0$ and $m=1.0$. The difference $\lambda_{(n+1)^{\prime}}-\lambda_{n^{\prime}}$ is not order $O\left(1 / N_{B S}\right)$ but order $O(1)$.

value of $N_{B S}$ is changed, while the differences $\lambda_{n^{\prime}}-\lambda_{m^{\prime}}$ are never of such order. This clearly shows the existence of the discrete spectrum $\left\{\lambda_{n^{\prime}}\right\}$.

Finally, we note other choices for the running coupling. If we tune $\lambda$ to an appropriate value, say $\lambda_{0}$, we obtain the solution of the HBS equation which describes the bound states in any system with different gauge group and matter contents. This is realized when we use the improved ladder and constant fermion mass approximations simultaneously. When we chose other gauge groups and matter content than $S U(3)$ and $N_{f}=3$, the parameter $\alpha_{0}\left(N_{c}=N_{f}=3\right) \equiv 4 \pi / 9$ defined in eq. (4.1) is modified. Since $\alpha_{0}$ is just an over overall constant in the right-hand-side of eq. (3.2), we can identify

$$
\lambda=\frac{9}{4 \pi} \alpha_{0}
$$

and we regard that all figures 3 , 6 and 5 shows the spectrum of $\alpha_{0}\left(M_{V}\right)$ scaled by $4 \pi / 9$. Figure 3 shows that for the case $2.23<\lambda_{0}<6.66$ the equation $\lambda_{1^{\prime}}\left(M_{V}\right)=\lambda_{0}$ has no solution, i.e., the level annihilation occurs and the ground state mass $M_{V}^{(1)}$ is given by $\lambda_{2^{\prime}}\left(M_{V}^{(1)}\right)=\lambda_{0}$. For the case $6.66<\lambda_{0}<8.22 M_{V}^{(1)}$ is given by $\lambda_{3^{\prime}}\left(M_{V}\right)=\lambda_{0}$. 
[The condition $\lambda_{0}>2.23$ corresponds to $N_{f}>10$ for $N_{c}=3$.]

\subsection{Bound state mass}

Using the method explained in section 3, we evaluate the mass of the vector bound state. We consider both the fixed and the running coupling cases with constant fermion mass. The vector bound state in weak fixed coupling case corresponds to the orthopositronium, the system of which is well-known both theoretically and experimentally 25]. We can explicitly check the validity of our method in this system. Next, we proceed to the running coupling case.

In the weak coupling limit the HBS equation for the orthopositronium is solved, and we obtain the famous non-relativistic result: 23, 26]

$$
M_{V}^{(n)}=2 m-\frac{m \alpha^{2}}{4 n^{2}},
$$

where $m$ is the pole mass of the fermion and antifermion. On the other hand, the spectrum of $\lambda$ (or $\lambda \alpha$ ) for a small binding energy in eq. (3.2) is given by [20]

$$
\lambda_{n^{\prime}}\left(M_{V}\right)=\frac{2 n}{\alpha} \sqrt{\frac{2 m-M_{V}}{m}} .
$$

Obviously, imposing $\lambda_{n^{\prime}}\left(M_{V}\right)=1$ in eq. (4.4) we obtain the non-relativistic result (4.3). The smallest eigenvalue $\lambda_{1^{\prime}}$ corresponds to the ground state $(n=1)$, and the second smallest eigenvalue $\lambda_{2^{\prime}}$ corresponds to the first excited state $(n=2)$, and so on. Furthermore, the binding energy and BS amplitude have no dependence on the gauge parameter. 27] [1

Here, we rescale all the dimensionful quantities so as to satisfy the relation $m \alpha=1$ fixing the coupling constant as $\alpha=1 / 137$ when we solve the HBS equation for the positronium. When the fermion mass becomes small, the support of the $T^{\prime}$-norm begins to shift to the infrared region. This is the reason why we use large value of the fermion mass compared with the unit scale.

We check whether the numerical calculation reproduce the relation (4.3) in the weak coupling limit. The spectrum (4.4) tells us that the quantity $\lambda_{1^{\prime}} / \lambda_{n^{\prime}}$ should be an integer. We adjust the bound state mass $M_{V}$ as $\lambda_{1^{\prime}}\left(M_{V}\right)=1$ : we update $M_{V}$ until the difference $\delta M_{V}$ given in eq. (3.8) satisfies $\left|\delta M_{V}\right|<m \alpha^{4}$. We show the spectrum of the eigenvalues $\lambda_{n^{\prime}}$ with $N_{B S}=11,14$ and 17 in table 2 . The results show that

$\|$ In the fixed strong coupling and constant mass the positronium does not exist and there are only continuum spectrum and no discrete spectrum. 20] In the case of the vanishing center-of-mass momentum $q_{\mu}=0$ the HBS equation is solved and a strong dependence on the gauge parameter is observed.28 


\begin{tabular}{|c||c|c|c|}
\hline$N_{B S}$ & 11 & 14 & 17 \\
\hline$\lambda_{1^{\prime}} / \lambda_{2^{\prime}}$ & 2.12 & 2.10 & 2.09 \\
$\lambda_{1^{\prime}} / \lambda_{3^{\prime}}$ & 3.13 & 3.16 & 3.17 \\
$\lambda_{1^{\prime}} / \lambda_{4^{\prime}}$ & 3.37 & 3.32 & 3.29 \\
$\lambda_{1^{\prime}} / \lambda_{5^{\prime}}$ & 4.36 & 4.39 & 4.38 \\
\hline
\end{tabular}

Table 2: The spectrum of eigenvalues $\lambda_{n^{\prime}}$ for $N_{B S}=11,14$ and 17 when the bound state mass is adjusted such that the largest eigenvalue $\lambda_{1^{\prime}}$ is equal to unity. This should be compared with the result in the non-relativistic limit, $\lambda_{1^{\prime}} / \lambda_{n^{\prime}}=n$.

$\lambda_{1^{\prime}} / \lambda_{n^{\prime}}$ appears as an integer for the first several $n$ 's within about $10 \%$ errors. As for the quantum number $n^{\prime}$ larger than five, $\lambda_{1^{\prime}} / \lambda_{n^{\prime}}$ suffers large numerical errors. This simply comes from the fact that we do not cover the supports of such higher radial excited states. We calculate the binding energies of the first three states. The binding energy is defined by $B^{(n)}=2 m-M_{V}^{(n)}$ and is given in table 3 with $N_{B S}=11$, 14 and 17. This result should be compared with that in the non-relativistic limit:

\begin{tabular}{|c||c|c|c|}
\hline$N_{B S}$ & 11 & 14 & 17 \\
\hline$B^{(1)}[\mathrm{Ryd}]$ & 0.513 & 0.489 & 0.479 \\
$B^{(2)}[\mathrm{Ryd}]$ & 0.121 & 0.116 & 0.115 \\
$B^{(3)}[\mathrm{Ryd}]$ & 0.0544 & 0.0515 & 0.0505 \\
\hline
\end{tabular}

Table 3: The biding energies for the ground state $\left(B^{(1)}\right)$, the first excited state $\left(B^{(2)}\right)$ and the second excited state $\left(B^{(3)}\right)$ with $N_{B S}=11,14$ and17. The values are in the units of the Rydberg energy, $m \alpha^{2} / 2$.

$B^{(n)}[\operatorname{Ryd}]=1 /\left(2 n^{2}\right)(=0.5,0.125,0.0556, \ldots)$, and shows that our method works well.

Now that, we proceed to the running coupling case. We update the bound state mass $M_{V}$ until the difference $\delta M_{V}$ given in eq. (3.8) satisfies $\left|\delta M_{V}\right|<10^{-2}$. As studied in section 4.1, the ground state mass is given by the solution of $\lambda_{1^{\prime}}\left(M_{V}\right)=1$ for $0.5<m$ and by $\lambda_{2^{\prime}}\left(M_{V}\right)=1$ for $0.16<m<0.5$. In the latter case, the ground and first excited states are given by $\chi_{2^{\prime}}$ and $\chi_{3^{\prime}}$, respectively, due to the level annihilation. We show the binding energies $\left(B^{(n)} \equiv 2 m-M_{V}^{(n)}\right)$ of the ground 
and first excited states for various fermion masses in fig. 6. This figure shows that

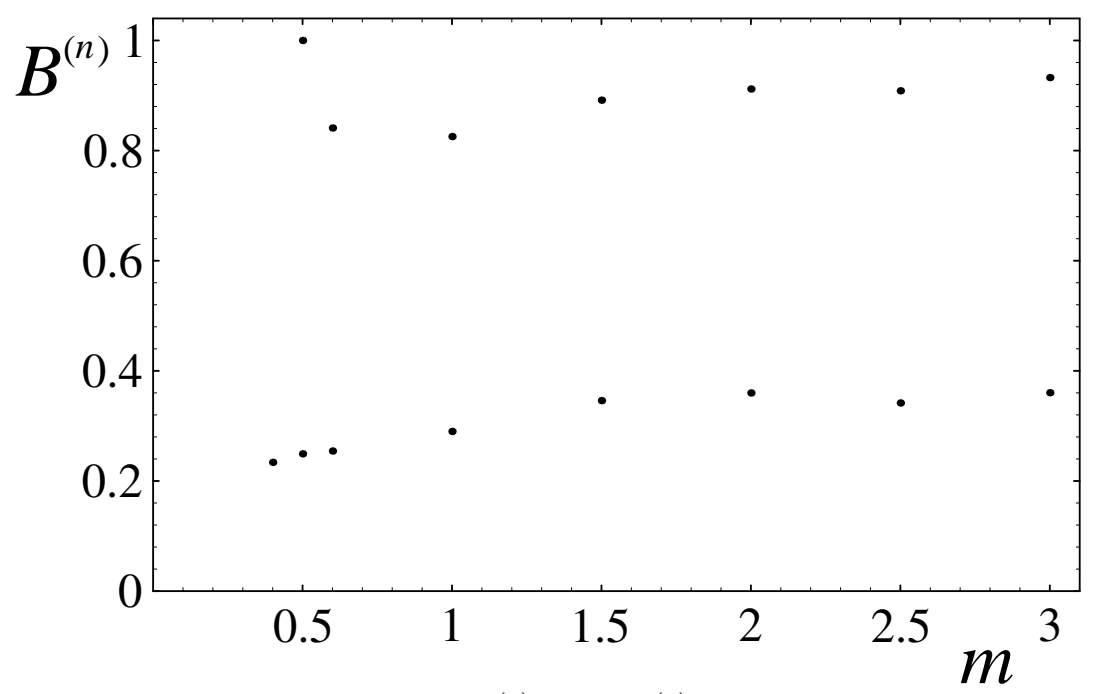

Figure 6: The binding energies $B^{(1)}$ and $B^{(2)}$ for various constant fermion masses. The upper half data are of $B^{(1)}$ and the lower half are of $B^{(2)}$.

$B^{(1)}=2 m$ at $m=0.5$, i.e., $\chi_{1^{\prime}}$ is massless state. At $m=0.4$ the state $\chi_{1^{\prime}}$ and its eigenvalue $\lambda_{1^{\prime}}$ have no physical correspondence due to the level annihilation, and the binding energy of only the ground state is calculated from $\lambda_{2^{\prime}}\left(M_{V}\right)=1$. As a result, the binding energy of the ground state changes discretely from $1.0(=2 \mathrm{~m})$ to 0.25 around $m \sim 0.5$. We make some comments here. The binding energies $B^{(1)}$ and $B^{(2)}$ approach to the values $B^{(1)} \rightarrow 1$ and $B^{(2)} \rightarrow 0.4$ asymptotically for large fermion mass. It is plausible that the binding energies of a heavy bound states do not depend on the mass of their heavy constituents thanks to the decoupling theorem 29]. We may also read off the values of fermion masses with which we obtain massless bound states from fig. 5 .

\section{Discussions}

As discussed in the Introduction, ideally we are better to solve the SD equation in the same approximation as the BS equation in order to obtain the full propagator (or mass function). For the purpose that we study the numerical method for solving the HBS equation for the massive bound state, we use the constant, i.e., tree level, mass $\Sigma(x)=m$ in this paper. It is true that this approximation is good in the weak coupling QED and retains the non-relativistic result. For applying our method to the real QCD case, however, we should use the mass function in the fermion propagator. 
Even when we consider the heavy quarkonia, the quantum corrections to the mass function have the same order as the binding energy. Especially in the case of the light quarkonia ( $\rho$ meson) the effect of the mass function becomes more important. As a result, the running effect of mass function is always important for any quarkonia, and is same order of magnitude as the binding force.

In the real QCD there exists the states with negative binding energy (for highly excited states) because of the confining potential. Our choice of the running coupling does not generate the confining force, while a suitable form of the running coupling generates it. The non-relativistic quark model with the Richardson potential gives a good result for the low-lying spectrum of the heavy quarkonia.[30] In the constant mass approximation the $q \bar{q}$ free quark threshold opens at $q^{2}=(2 m)^{2}$. ${ }^{\text {* }}$ Thus the states with negative binding energy are unstable. We observe that infinitely many number (the order of total lattice points) of states are lying in the region $M_{V}<2 m$. We consider only the states which have positive binding energy. Only a first few state will be relevant in the constant mass approximation.

In this paper, we study the the solutions of the homogeneous Bethe-Salpeter equation in the constant mass and improved ladder approximations. We leave the application of our method to the quarkonia for future work.

\section{Appendix}

\section{A Schwinger-Dyson Equation}

In this appendix, we briefly review how to derive the fermion mass function in the complex plane. Here, following ref. [19], we perform the analytic continuation to the $\mathrm{SD}$ equation in the integral form.

In order to solve the Bethe-Salpeter (BS) equation we need a fermion propagator $S_{F}(p)$, which is determined by the Schwinger-Dyson (SD) equation. When the ladder approximation is adopted for the BS equation, it is natural to use the same approximation for the SD equation. In the strong interaction the mass function of the quark propagator receives large quantum correction of the same order $\Lambda_{\mathrm{QCD}}$ as that of binding force.

\footnotetext{
** If we take into account of the running effect of the mass function by solving the SD equation, the resultant quark propagator has no pole for the real momentum. [17, 18
} 
The SD equation in the (improved) ladder approximation is given by

$$
i S_{F}^{-1}(p)=\not p-m-\int \frac{d^{4} k}{(2 \pi)^{4} i} C_{2} g^{2}(p, k) \frac{1}{-l^{2}}\left(g_{\mu \nu}-\frac{l_{\mu} l_{\nu}}{l^{2}}\right) \gamma^{\mu} i S_{F}(k) \gamma^{\nu}
$$

where $l_{\mu}=(p-k)_{\mu}, C_{2}=\left(N_{c}^{2}-1\right) /\left(2 N_{c}\right)$ is a second Casimir invariant and $m$ is the bare mass of the fermion. The Landau gauge is adopted for the propagator of the gauge boson. When we consider QCD, we use the running coupling in the SD equation (A.1). Here for definiteness we adopt the Higashijima-Miransky approximation 21, 22 to the running coupling, i.e., $g^{2}(p, k)=g^{2}\left(\max \left(-p^{2},-k^{2}\right)\right)$. In considering QED we use fixed coupling $g^{2}(p, k)=e^{2}$ and define $C_{2}=1$.

In general the fermion propagator is expanded by two scalar functions $A$ and $B$ :

$$
i S_{F}^{-1}(p)=A\left(-p^{2}\right) \not p-B\left(-p^{2}\right) \text {. }
$$

The mass function $\Sigma(z)$ is defined by $\Sigma(z)=A(z) / B(z)$. Substituting eq. (A.2) into the SD equation (A.1) we find $A(z)=1$ and

$$
\Sigma(z)=m+\frac{3 C_{2}}{16 \pi^{2}} \int_{0}^{\infty} y d y \frac{g^{2}(\max (z, y))}{\max (z, y)} \frac{\Sigma(y)}{y+\Sigma^{2}(y)} .
$$

Now, let us discuss the problem i) stated in sec. 1. When we solve the homogeneous $\mathrm{BS}$ equation for the massive bound state, we have to use the mass function on the complex plane. The mass function $\Sigma(z)$ is needed along the set of parabolic curves $z=\left(u \mp i M_{V} / 2\right)^{2}+x^{2}$, where $M_{V}$ is the mass of the bound state. We can restrict ourselves to the case $\operatorname{Im} z>0$ because of $\Sigma(z)^{*}=\Sigma\left(z^{*}\right)$. It is not difficult to calculate such a mass function. In refs. [17, 18] the SD equation (A.3) is converted into the differential equation for carrying out the analytic continuation of the mass function. On the other hand, we can easily solve the SD equation (A.3) as the integral equation itself by the iteration. [19] After carrying out the analytic continuation, the $\mathrm{SD}$ equation becomes

$$
\begin{aligned}
\Sigma(z)= & +\frac{3 C_{2}}{16 \pi^{2}}\left[\int_{0}^{-M_{V}^{2} / 4} d y \frac{y g^{2}(z)}{z}\right. \\
& \left.+\int_{C\left(-M_{V}^{2} / 4, z\right)} d y \frac{y g^{2}(z)}{z}+\int_{C(z, \infty)} d y g^{2}(y)\right] \frac{\Sigma(y)}{y+\Sigma^{2}(y)},
\end{aligned}
$$

where $C(a, b)$ is the contour from the point $a$ to $b$ and is lying on the parabolic curve with $\operatorname{Im} z>0$. To say more completely, we should take account of the singularity of the running coupling. We can solve the SD equation (A.4) by the iteration on the parabolic curve as usual. Then, we obtain the mass function on the complex plane from eq. (A.4). 


\section{Acknowledgments}

We would like to thank Taichiro Kugo and Joseph Schechter for helpful discussions and comments. We also thank Ken Nakanishi for a comment.

\section{References}

[1] Y. Nambu, Prog. Theor. Phys. 5, 614 (1950).

[2] E.E. Salpeter and H.A. Bethe, Phys. Rev. 84, 1232 (1951).

[3] See, for a review, N. Nakanishi Prog. Theor. Phys. Suppl. 43, 1 (1969), see also, N. Nakanishi, in Proc. of 1991 Nagoya Spring School of Dynamical Symmetry Breaking, Apr. 23-27, 1991, ed. K. Yamawaki (World Scientific Pub. Co., Singapore, 1991).

[4] K.-I. Aoki, M. Bando, T. Kugo, M.G. Mitchard and H. Nakatani, Prog. Theor. Phys. 84, 683 (1990).

[5] P. Jain and H.J. Munczek, Phys. Rev. D 44, 1873 (1991).

[6] T. Kugo, M.G. Mitchard and Y. Yoshida, Prog. Theor. Phys. 91, 521 (1994).

[7] Y. Nambu, Phys. Rev. Lett. 4 (1960) 380; Phys. Rev. 117, 648 (1960).

[8] J. Goldstone, Nuovo Cim. 19, 154 (1961);

J. Goldstone, A. Salam and S. Weinberg, Phys. Rev. 127, 965 (1962).

[9] T. Maskawa and H. Nakajima, Prog. Theor. Phys. 52, 1326 (1974); ibid. 54, 860 (1975).

[10] T. Kugo and M.G. Mitchard, Phys. Lett. B 282, 162 (1992); ibid. 286, 355 (1992).

[11] M. Bando, M. Harada and T. Kugo, Prog. Theor. Phys. 91, 927 (1994).

[12] K.-I. Aoki, T. Kugo and M.G. Mitchard, Phys. Lett. B 226, 467 (1991).

[13] M. Harada and Y. Yoshida, Phys. Rev. D 50, 6902 (1994).

[14] E.E. Salpeter, Phys. Rev. 87, 328 (1952).

[15] H.J. Munczek and P. Jain, Phys. Rev. D 46, 438 (1992). 
[16] P. Jain and H.J. Munczek, Phys. Rev. D 48, 5403 (1993).

[17] R. Fukuda and T. Kugo, Nucl. Phys. B117, 250 (1976).

[18] D. Atkinson and D.W.E. Blatt, Nucl. Phys. B151, 342 (1979).

[19] T. Kugo and Y. Yoshida, talk given at molecule type workshop "SchwingerDyson equation and Bethe-Salpeter equation" in 1994, Yukawa Institute of Theoretical Physics in Kyoto, to appear in Soryuushiron Kenkyu (in Japanese); Y. Yoshida, Ph. D thesis, "Chiral Symmetry, Heavy Quark Symmetry and Bound States".

[20] See, for a review, N. Setô, Prog. Theor. Phys. Suppl. 95, 25 (1988).

[21] K. Higashijima, Phys. Rev. D 29, 1228 (1984).

[22] V.A. Miransky, Sov. J. Nucl. Phys. 38, 280 (1984).

[23] G. Tiktopoulos, J. Math. Phys. 6, 573 (1965).

[24] L.G. Suttorp, Ann. of Phys. 113, 257 (1978).

[25] See, for example, T. Murota, Prog. Theor. Phys. Suppl. 95, 46 (1988).

[26] See, for example, C. Itzykson and J.-B. Zuber, "Quantum Field Theory", (McGraw-Hill, New York, 1980).

[27] I. Fukui, N. Setô and T. Yoshida, Prog. Theor. Phys. 58, 1585 (1977).

[28] A. Nishimura and K. Higashijima, Prog. Theor. Phys. 56, 908 (1976).

[29] T. Appelquist and J. Carazzone, Phys. Rev. D 11, 2856 (1975).

[30] J.L. Richardson, Phys. Lett. 82B, 272 (1979). 